\title{
New Stone-Weierstrass Theorem
}

\author{
Hueytzen J. Wu \\ Department of Mathematics, Texas A\&M University-Kingsville, Kingsville, USA \\ Email: hueytzen.wu@tamuk.edu
}

How to cite this paper: $\mathrm{Wu}$, H.J. (2016) New Stone-Weierstrass Theorem. Advances in Pure Mathematics, 6, 943-947. http://dx.doi.org/10.4236/apm.2016.613071

Received: September 25, 2016

Accepted: December 9, 2016

Published: December 12, 2016

Copyright $\odot 2016$ by author and Scientific Research Publishing Inc. This work is licensed under the Creative Commons Attribution International License (CC BY 4.0).

http://creativecommons.org/licenses/by/4.0/

(c) (7) Open Access

\begin{abstract}
Without the successful work of Professor Kakutani on representing a unit vector space as a dense vector sub-lattice of $(\boldsymbol{C}(\boldsymbol{X}),\|\cdot\|)$ in 1941, where $\boldsymbol{X}$ is a compact Hausdorff space and $\boldsymbol{C}(\boldsymbol{X})$ is the space of real continuous functions on $\boldsymbol{X}$. Professor M. H. Stone would not begin to work on "The generalized Weierstrass approximation theorem" and published the paper in 1948. Latter, we call this theorem as "Stone-Weierstrass theorem" which provided the sufficient and necessary conditions for a vector sub-lattice $\boldsymbol{V}$ to be dense in $(\boldsymbol{C}(\boldsymbol{X}),\|\cdot\|)$. From the theorem, it is not clear and easy to see whether 1) "the vector sub-lattice $V$ of $C(X)$ contains constant functions" is or is not a necessary condition; 2) Is there any clear example of a vector sub-lattice $\boldsymbol{V}$ which is dense in $(\boldsymbol{C}(\boldsymbol{X}),\|\cdot\|)$, but $\boldsymbol{V}$ does not contain constant functions. This implies that we do need some different version of "Stone-Weierstrass theorem" so that we will be able to understand the "Stone-Weierstrass theorem" clearly and apply it to more places where they need this wonderful theorem.
\end{abstract}

\section{Keywords}

Compact Hausdorff Space, Vector Sub-Lattice, Vector Sub-Algebra, Stone-Weierstrass Theorem

\section{Introduction}

Throughout this paper, $[\boldsymbol{T}]^{<\omega}$ denotes the collection of all finite subsets of the given set $T$, "nhood" represents the word "neighborhood", $C(Z)$ (or $C(X)$ ) is the space of real (or bounded real) continuous functions on compact Hausdorff space $Z$ (or $X$ ), and $\|\cdot\|$ is the supremum norm; i.e., $\|f\|=\operatorname{Sup}\{\mid f(x) \| x \in Z($ or $X)\}$. For the other terminologies in Functional Analysis or General Topology which are not explicitly defined in this paper, the readers will be referred to the References [1] [2].

Works on the sufficient and necessary conditions for a vector sub-lattice or vector 
sub-algebra $V$ to be dense in $(C(Z),\|\cdot\|)$ were initiated in 1941 when Professor Kakutani tried to represent an order unit space $V$ as a dense vector sub-lattice of $(\boldsymbol{C}(\mathbf{Z}),\|\cdot\|)$. It seemed that at that time Professor Kakutani did not know the sufficient and necessary conditions for a vector sub-lattice $\boldsymbol{V}$ to be dense in $(\boldsymbol{C}(\mathbf{Z}),\|\| \|)$. But it is clear that Professor Kakutani knew that a vector sub-lattice $V$ is dense in $(\boldsymbol{C}(\boldsymbol{Z}),\|\cdot\|)$ if 1$) \boldsymbol{V}$ separates points of $Z$; and 2) $V$ contains constant functions. In 1948, when Professor M. H. Stone published the "Generalized Weierstrass approximation theorem", as I know, he did give honor and credit to Professor Kakutani for the work in inspiring the paper M. H. Stone published in 1948. In my personal opinion, a) $V$ separates points of $Z$ and b) $V$ contains constant functions are sufficient conditions for a vector sub-lattice $V$ to be dense in $(\boldsymbol{C}(\boldsymbol{Z}),\|\cdot\|)$. It seemed that it first appeared in Professor Kakutani's paper in1941. So, we should call this theorem as "Kakutani's theorem". Therefore, we will cite the Theorem 3.4 as Kakutani's Theorem in Section 3 and prove it with the results either in Section 2 or in a Theorem of Section 4.

\section{A Characterization of Compact Sets}

Due to the lack of original document in proving $X$ in Section 3 is compact by Professor Kakutani. We insert this section as Section 2 to develop some necessary results for proving that $\boldsymbol{X}$ is a compact Haudorff space. Let $\boldsymbol{A}$ be a family of continuous functions on a topological space $\boldsymbol{Y}$. A net $\left\{x_{i}\right\}$ in $\boldsymbol{Y}$ is called an $A$-net if $\left\{f\left(x_{i}\right)\right\}$ converges for all $\mathrm{f}$ in $A$.

Proposition 2.1 Let $A=\left\{f_{\alpha} \mid \alpha \in \Lambda\right\}$ be a family of continuous functions $f_{\alpha}$ on $Y$ into Hausdorff spaces $Y_{\alpha}$ such that the topology on $Y$ is the weak topology induced by A. $\boldsymbol{E}, \boldsymbol{F}$ two subspaces of $\boldsymbol{Y}$ such that $\boldsymbol{E} \subseteq \boldsymbol{F} \subseteq \mathrm{Cl}(\boldsymbol{E})$, where $\mathrm{Cl}(\boldsymbol{E})$ is the closure of $\boldsymbol{E}$ in $Y$. Then the following are equivalent. 1) Every $A$-net in $E$ has a cluster point in F. 2) Every $A$-net in F converges in $F$.

Proof. Let $\left\{y_{j}\right\}$ be an $A$-net in $F$. For each $y_{j}$, pick a net $\left\{x_{i}^{j}\right\}$ in E converging to $y_{j}$. For each $f_{\alpha}$ in $A,\left\{f_{a}\left(y_{j}\right)\right\}$ converges to a point $z_{\alpha}$ in $Y_{\alpha}$ and $\lim \left\{f_{a}\left(x_{i}^{j}\right)\right\}=f_{a}\left(y_{j}\right)$, for each open nhood $O_{\alpha}^{t}$ of $z_{\alpha}$, there is an $x_{\alpha}^{t}$ in $E$ such that $f_{a}\left(x_{\alpha}^{t}\right) \in O_{\alpha}^{t}$. Thus for any $H \in L^{<w}$ and $O_{H}^{t}=\left\{O_{\alpha}^{t} \mid O_{\alpha}^{t}\right.$ is an open nhood of $z_{\alpha}, \alpha$ is in $\left.H\right\}$, there is an $x_{H}^{t}$ in $E$ such that $f_{a}\left(x_{H}^{t}\right)$ is in $O_{\alpha}^{t}$ for each $\alpha$ in $H$. Direct $\left\{(H, t) \mid H \in \Lambda^{<w}, O_{\alpha}^{t}\right.$ is an open nhood of $\left.z_{a}, a \in H\right\}$ by setting that $\left(H_{1}, t_{1}\right) \geq\left(H_{2}, t_{2}\right)$ iff $H_{1} \supset H_{2}$ and $O_{\alpha}^{t_{1}} \subset O_{\alpha}^{t_{2}}$ for each $\alpha$ in $H_{2}$. Then $\left\{x_{H}^{t}\right\}$ is an A-net in $E$. (1) implies that $\left\{x_{H}^{t}\right\}$ has a cluster point $x$ in $F$. Since $Y_{\alpha}$ is Hausdorff and $\left\{f_{a}\left(x_{H}^{t}\right)\right\}$ converges for each $\alpha$ in $\Lambda$, thus $\left\{f_{a}\left(x_{H}^{t}\right)\right\}$ converges to $f_{a}(x)$ for each $\alpha$ in $\Lambda$. This implies that $\lim \left\{f_{a}\left(y_{j}\right)\right\}=f_{a}(x)$ for each $\alpha$ in $\Lambda$. Thus $\left\{y_{j}\right\}$ converges to $x$ in $F$. (2) implying (1) is obvious.

Theorem 2.2 Let $A$ be a family of continuous functions on a topological space $Y$. Then $Y$ is compact iff 1) $f(Y)$ is contained in a compact subset $C_{f}$ for each $f$ in $A$, and 2) every A-net has a cluster point in $Y$.

Proof. Let $\left\{x_{i}\right\}$ be an ultranet in $Y$. For each $\mathrm{f}$ in $A,\left\{f\left(x_{i}\right)\right\}$ is an ultranet in $C_{f}$ hence converges in $C_{b}$ i.e., $\left\{x_{i}\right\}$ is an A-net. (2) implies that $\left\{x_{i}\right\}$ has a cluster point 
$\mathrm{x}$ in $Y$. Since $\left\{x_{i}\right\}$ is an ultranet, $\left\{x_{i}\right\}$ converges to $x$. Thus, $Y$ is compact. The converse is obvious.

Corollary 2.3 Let $A$ be a family of continuous functions on $Y$ into Hausdorff spaces such that the topology on $\boldsymbol{Y}$ is the weak topology induced by A. $E$ a subspaces of $\boldsymbol{Y}$ then $\mathrm{Cl}(E)$ is compact iff 1$) \mathrm{Cl}(f(E))$ is compact for each $f$ in $A$, and 2) every $A$-net in $E$ converges in $\mathrm{Cl}(E)$.

\section{Kakutani Theorem}

Definition 3.1 An element $\mathrm{e}$ in a vector lattice $V$ is called an order unit if for every $V$ in $V$, there is a $r>0$ such that $|\mathrm{v}| \leq$ re.

Definition 3.2 A topological vector lattice $V$ is called an order unit space if $V$ contains an order unit e such that the topology on $V$ is equivalent to the topology induced by the unit norm $\|v\|_{e}=\operatorname{Inf}\{r>0 \| v \mid \leq r e\}$ for every $v$ in $V$.

Let $L$ be the collection of all real continuous lattice homomorphisms t on the order unit space $\left(\boldsymbol{V},\|\|_{e}\right)$. Equip $L$ with the weak topology induced by $V$. Then $V$ is a space of real continuous functions on $L$. From now on, we regard every $V$ in $V$ as a real continuous function on $L$ defined by $v(t)=t(v)$ for all $t$ in $L$. It is obvious that:

1) $V$ separates points of $L$ : Since for any two different points $s$ and $t$ in $L$, $s$ and $t$ are two different real continuous lattice homomorphisms on $\left(V,\|\cdot\|_{e}\right)$, thus there is a $V$ in $V$ such that $s(v) \neq \boldsymbol{t}(v)$; i.e. $v(s) \neq v(\boldsymbol{t})$. This implies that $V$ separates points of $\boldsymbol{L}$.

2) $L$ is a Hausdorff space: Since the topology on $L$ is the weak topology induced by $V$, $V$ is a set of real continuous functions on $L$ and $V$ separates point of $L$, therefore, $L$ is a Hausdorff space. Let $X=\{t \in \boldsymbol{L} \mid t(e)=1\}$, then $\boldsymbol{X}$ is a Hausdorff space. I believe that Professor Kakutani had proved that $X$ is compact. We have no document available to see his proof. Let's prove it by Corollary 2.3 in this paper as the following:

Theorem $3.3 X$ is a compact Hausdorff space.

Proof. By the setting, $X=e^{-1}(\{1\})$, then $X$ is closed; i.e., $\mathrm{Cl}(X)=X$. Let's prove that $X$ satisfies (1) and (2) in Corollary 2.3: 1) For each $v$ in $V$, there is a $\mathrm{n}$ in $\mathbb{N}$ such that $|v|<n e$, thus $|v|(x)<n e(x)=n$; i.e. $\operatorname{Cl}\{v(x) \mid x$ is in $X\} \subseteq[-n, n]$. So,

$\operatorname{Cl}\{v(x) \mid x$ is in $X\}$ is compact. 2) Let $\left\{x_{i}\right\}$ be a $V$-net in $X$. Let $r$ be the function from $V$ to $\mathbb{R}$ defined by $r(v)=\lim \left\{v\left(x_{i}\right)\right\}$, it can be readily proved that $r$ is a real lattice homomorphism on $V$ such that $r(e)=1$; i.e. $r$ is in $X$ and $\left\{x_{i}\right\}$ converges to $r$. By Corollary 2.3 $X=\mathrm{Cl}(\boldsymbol{X})$ is compact.

Next, we will use the result of Stone-Weierstrass theorem (Theorem 4.1) to prove that $V$ is dense in $(\boldsymbol{C}(\boldsymbol{X}),\|\|)$.

\section{Kakutani's Theorem}

Theorem 3.4 Let $V$ be a vector sub-lattice of $C(X)$ such that 1) $V$ separates points of $X$, and 2) $V$ contains constant functions, then $V$ is dense in $(\boldsymbol{C}(\boldsymbol{X}),\|\|$.$) .$

Proof. We are going to show that for any $\mathrm{f}$ in $C(X)$, any $x, y$ in $X$ and any $\varepsilon>0$, there is a $g$ in $V$ such that $|f(x)-g(x)|<\varepsilon$ and $|f(y)-g(y)|<\varepsilon$ : For any $x$ and $y$ in $X$, since $\boldsymbol{V}$ separates points of $\boldsymbol{X}$, pick a $k$ in $\boldsymbol{V}$ such that $k(x) \neq k(y)$. Then 
$\alpha+\beta k(x)=f(x)$ and $\alpha+\beta k(y)=f(y)$ has a unique pair of solutions for $\alpha$ and $\beta$. Since $V$ contains constant function, let $g=\alpha+\beta k$, then $g$ is in $V$ such that $|f(x)-g(x)|=0<\varepsilon$ and $|f(y)-g(y)|=0<\varepsilon$. By the Stone-Weierstrass theorem, $V$ is dense in $(\boldsymbol{C}(\boldsymbol{X}),\|\cdot\|)$.

\section{Notes:}

1) A lot of textbooks of Functional Analysis listed The above theorem as the "StoneWeierstrass Theorem". I strongly disagree on it.

2) In my opinion, the above Theorem 3.4 should be named as Kakutan's theorem. Because Professor Kakutani used the result of this theorem to represent an order unit space $\left(\boldsymbol{V},\|\|_{e}\right)$ as a dense subspace of $(\boldsymbol{C}(\boldsymbol{X}),\|\| \|)$, where $\boldsymbol{X}$ is compact Hausdorff space.

\section{A New Version of Stone-Weierstrass Theorem for $(C(X),\|\| \|)$}

Due to that the closure of a sub-algebra is a vector sub-lattice of $C(X)$ (by Lemma 44.3 in the Reference [1], p. 291), therefore, the sufficient and necessary conditions for a vector sub-lattice $V$ of $\boldsymbol{C}(\boldsymbol{X})$ to be dense in $(\boldsymbol{C}(\boldsymbol{X}),\|\cdot\|)$ are also the sufficient and necessary conditions for a vector sub-algebra of $\boldsymbol{C}(\boldsymbol{X})$ to be dense in $(\boldsymbol{C}(\boldsymbol{X}),\|\cdot\|)$. Let's cite "The generalized Wierstrass approximation theorem" in the Reference ([3], p, 170) as the Theorem 4.1 in the following:

\section{Theorem 4.1. Stone-Weierstrass Theorem}

Let $\mathbf{Z}$ be a compact Hausdorff space. A vector sub-lattice or a sub-algebra $V$ of $C(Z)$ is dense in $(\boldsymbol{C}(\boldsymbol{Z}),\|\| \|)$ iff 1$) V$ separates points of $Z$, and 2) for any $f$ in $C(Z)$, any $x, y$ in $Z$, and any $\varepsilon$ with $0<\varepsilon<1$, there is a $g$ in $V$ such that $|f(x)-g(x)|<\varepsilon$ and $|f(y)-g(y)|<\varepsilon$.

\section{Theorem 4.2. New Version of Stone-Weierstrass}

\section{Theorem}

Let $\mathbf{Z}$ be a compact Hausdorff space. A vector sub-lattice or sub-algebra $V$ of $C(Z)$ is dense in $(\boldsymbol{C}(\mathbf{Z}),\|\|)$ iff 1$) V$ separates points of $Z$, and 2) for any $x$ in $Z$, and any $\varepsilon$ with $0<\mathcal{E}<1$, there is a $g$ in $V$ such that $1-g(x) /\|g\|<\varepsilon$.

To show the equivalence between Theorem 4.1 and Theorem 4.2, it is enough to show the equivalence between the following statements (A) and (B):

(A) for any $f$ in $C(Z)$, any $x, y$ in $Z$ and any $\varepsilon$ with $0<\varepsilon<1$, there is a $g$ in $V$ such that $|f(x)-g(x)|<\varepsilon$ and $|f(y)-g(y)|<\varepsilon$.

(B) for any $x$ in $Z$ and any $\mathcal{E}$ with $0<\varepsilon<1$, there is a $g$ in $V$ such that $1-g(x) /\|g\|<\varepsilon$.

Proof. (A) $\Rightarrow$ (B): Let $h_{1}$ be the function in $C(Z)$ such that $h_{1}(x)=1$ for all $x$ in $Z$. Then for each $x$ in $Z$ and any $\varepsilon$ with $0<\varepsilon<1$, there is a $k_{x}$ in $V$ such that $\left|h_{1}(x)-k_{x}(x)\right|=|1-k x(x)|<\varepsilon / 2$; i.e. for any $\varepsilon$ with $0<\varepsilon<1,1-\varepsilon / 2<k_{x}(x)<1+\varepsilon / 2$. For each $x$ in $Z$, let $O_{x}=k_{x}^{-1}((1-\varepsilon / 2,1+\varepsilon / 2))$. Then $Z \subset \cup_{x \in Z} O_{x}$. Since $Z$ is compact, there exist $x_{1}, x_{2}, \cdots, x_{n}$ in $Z$ such that $Z \subseteq \cup\left\{O_{x_{i}}: i=1,2, \cdots, n\right\}$. Let $g=\operatorname{Sup}\left\{k x_{i} \mid i=1,2, \cdots, n\right\}$. Then $g$ is in $V$ and for all $x$ in $Z, 1-\varepsilon / 2 \leq g(x) \leq 1+\varepsilon / 2$. 
Thus $1-g(x) /\|g\|<1-(1-\varepsilon / 2) /(1+\varepsilon / 2)<\varepsilon$.

(B) $\Rightarrow$ (A): Let $f \in \boldsymbol{C}(\boldsymbol{Z})$. For every $\varepsilon>0$ and any two points $x, y$ in $Z$, let $k$ be in $V$ such that $k(x) \neq k(y)$. Without loss of generality, assume that there exist $\alpha, \beta$ in $\mathbb{R}$, $\alpha \neq 0$ such that $\alpha+\beta k(x)=f(x)$ and $\alpha+\beta k(y)=f(y)$. Pick $r, s$ in $V$ such that $1-r(x) /\|r\|<\varepsilon /|a|$ and $1-s(x) /\|s\|<\varepsilon /|a|$. Let $t=\operatorname{Sup}(r /\|r\|, s /\|s\|)$ and $g=\alpha t+\beta k$. Then $g$ is in $V$ satisfying that $|f(x)-g(x)|=(|\alpha|)|1-t(x)| \leq(|\alpha|)[1-r(x) /|r| \mid] \mid<\varepsilon$, and $|f(y)-g(y)|=(|\alpha|)|1-t(y)| \leq(|\alpha|)|[1-s(y) / \| s||]|<\varepsilon$.

Theorem 4.3. Theorem 4.1 and Theorem 4.2 are equivalent.

\section{Remark 4.4:}

1) If the vector sub-lattice or sub-algebra $V$ in the Theorem 3.4 contains constant functions, (without using Theorem 4.1) then let $g$ be the function such that $g(x)=1$ for all $x$ in $Z$, it is clear that the condition 2) in Theorem 4.2 is satisfied, thus by Theorem 4,2 , the vector sub-lattice or sub-algebra $V$ in Theorem 3.4 is dense in $(\boldsymbol{C}(\mathbf{Z}),\|\cdot\|)$.

2) It is also clear to get an example of a vector sub-lattice $V$ that is dense in $(C(Z),\|\|)$, but $V$ does not contain constant functions. See the example in the following:

Example. For each $t \in(0,1)$, let $e_{t}(x)=1$, if $x=t ; e_{t}(x)=(2 x-t) / t$, if $x \in[t / 2, t]$; $e_{t}(x)=(2 x-t-1) /(t-1)$, if $x \in[t,(t+1) / 2] ; e_{t}(x)=0$, otherwise. Let $e_{0}(x)=1$, if $x=0 ; e_{0}(x)=-2 x+1$, if $x \in[0,1 / 2] ; e_{0}(x)=0$, otherwise. Let $e_{1}(x)=1$, if $x=1$; $e_{1}(x)=2 x-1$, if $x \in[1 / 2,1] ; e_{1}(x)=0$, otherwise. Let $V$ be the vector sub-lattice of $\boldsymbol{C}([0,1])$ generated by $\left\{e_{t}: t \in[0,1]\right\}$. Then $V$ does not contain constant functions, and $V$ is dense in $(\boldsymbol{C}([0,1]),\|\cdot\|)$, by Theorem 4.2.

\section{Conclusions}

1) The Stone-Weierstrass Theorem is a great and wonderful theorem. We provided new version of Stone-Weierstrass Theorem, simply trying to understand the theorem better and trying to obtain more applications to where it should be.

2) It must be a tough work for getting sufficient and necessary conditions for a vector sub-lattice $\boldsymbol{V}$ to be dense in $(\boldsymbol{C}(\boldsymbol{X}),\|\| \|)$. It is not surprised for a mathematician to spend three years or more to get it. Recently, I heard that some publishers or editors of mathematical journals claimed that they could publish more than 50, 60, 80 or 90 multiauthors' papers in a year. Is it possible? Do you believe it? Any comments or information are welcome.

\section{References}

[1] Willard, S. (1970) General Topology. Addison-Wesley, Reading, MA.

[2] Schaefer, H.H. (1971) Topological Vector Spaces. Springer Verlag, New York. https://doi.org/10.1007/978-1-4684-9928-5

[3] Stone, M.H. (1948) The Generalized Weierstrass Approximation Theorem. Mathematics Magazine, 21, 167-184, 237-254. https://doi.org/10.2307/3029750 